ISSN 2452-5340

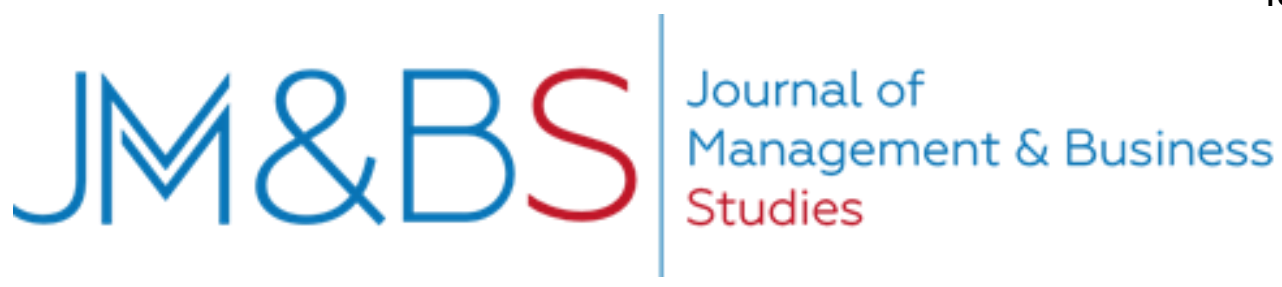

\title{
INCIDENCIA DE LOS TLC EN LAS PRÁCTICAS DE RESPONSABILIDAD SOCIAL DE LAS GRANDES EMPRESAS DE BARRANQUILLA ${ }^{1}$
}

\section{FTAS IMPACT ON THE SOCIAL RESPONSIBILITY PRACTICES OF THE BARRANQUILLA LARGE COMIPANIES}

\section{Camilo Mejía Reátiga ${ }^{2}$, Luz Agudelo Sánchez ${ }^{3}$, Marjorie Zúñiga Romero ${ }^{4}$, Javier Tous Chima ${ }^{5}$}

\section{Resumen}

Este artículo presenta los resultados del proyecto de investigación encaminado a determinar y describir la manera como los tratados de libre comercio suscritos por Colombia con Estados Unidos y Canadá, promueven la creación de prácticas de Responsabilidad Social en las grandes empresas.

Para tal efecto se examinaron aspectos fundamentales asociados al Pacto Global de las Naciones Unidas, desde una aproximación cualitativa por parte de las prácticas de las grandes empresas en la ciudad de Barranquilla en cuatro áreas temáticas: derechos humanos, derechos laborales, medio ambiente y lucha anticorrupción.

PALABRAS CLAVE: TLC, Pacto Global, Responsabilidad Social.

\footnotetext{
${ }^{1}$ El presente artículo es producto del proyecto de investigación "Incidencia de los tratados de libre comercio suscritos con Estados Unidos y Canadá en las prácticas de Responsabilidad Social de las grandes empresas de Barranquilla", en el marco del área de integración y comercio internacional. Financiación interna de la Universidad del Norte.

${ }^{2}$ Académico, Universidad del Norte, Barranquilla, Colombia. Ph.D. en Administración en el área de organizaciones, Universidad EAFIT, Colombia. cmejia@ uninorte.edu.co. https://orcid.org/0000-0002-8687-2250.

${ }_{3}^{3}$ Académico, Universidad del Norte, Barranquilla, Colombia. Magíster en Derecho Administrativo, Universidad Libre de Colombia, Colombia. Magíster en Derecho Medio Ambiental, Universidad Complutense de Madrid, España. leagudelo@uninorte.edu.co.

${ }^{4}$ Académico, Universidad del Norte, Barranquilla, Colombia. Doctor en Ciencias del Seguro, Universidad Pontifica de Salamanca, España. marjoriez@ uninorte.edu.co.

5 Académico, Universidad del Norte, Barranquilla, Colombia. Magíster en Derechos Humanos y Derecho Humanitario, Université Paris III, Francia. Magíster en Historia, Teoría y Práctica de los Derechos Humanos, Université Grenoble II - Pierre Mendes, Francia. Magíster en Prácticas de las Organizaciones Internacionales y Protección de los Derechos Humanos, Université Catholique de Lyon, Francia. tousj@uninorte.edu.co.
} 
ISSN 2452-5340

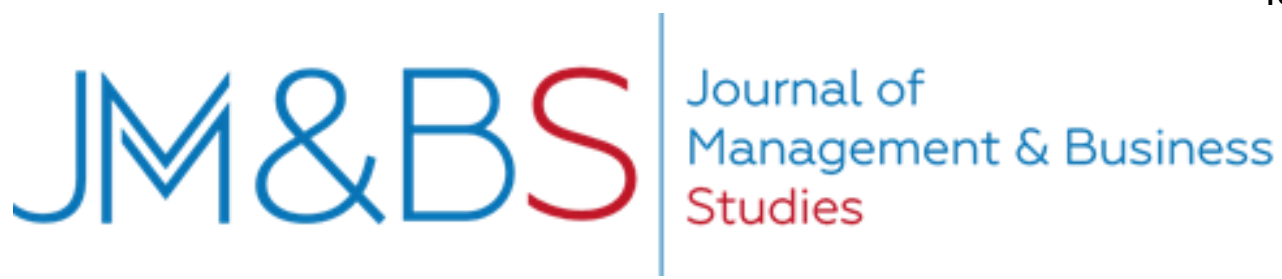

\begin{abstract}
This article presents the results of the research project aims to identify and describe the way the free trade agreements signed by Colombia with the United States and Canada, promoting the creation of social responsibility practices in large enterprises.

The paper examines key issues associated with the Global Compact of the United Nations, from a qualitative approach by the practices of large companies in the city of Barranquilla in four thematic areas such as human rights, labor rights, environment and anti-corruption.
\end{abstract}

KEYWORDS: FTA, Global Compact, Social Responsability.

\section{Introducción}

Según Bremer (2008), la globalización es un hecho de la vida que ha subestimado su fragilidad debido a la expansión de los mercados que superan la capacidad de las sociedades y de sus sistemas políticos para ajustarse a ellos. El intercambio de bienes y servicios como consecuencia de la globalización hizo que países emergentes como Colombia, iniciaran procesos de liberalización de mercados con la finalidad de permitir intercambio de bienes y servicios competitivos. En el desarrollo de estos procesos Colombia suscribió tratados de libre comercio, TLC con Canadá y Estados Unidos que entraron en vigencia durante los años 2011 y 2012 respectivamente.

Estos acuerdos comerciales no se limitan únicamente a regular el intercambio económico entre los estados firmantes, sino que exigen la creación de un marco de políticas que responda a un nuevo paradigma fundamentado en la responsabilidad social empresarial, como una nueva herramienta que ha de cambiar la visión de las relaciones entre la economía, la política y la sociedad civil (Ancos y Vicente, 2009, p.78).

$\mathrm{La}$ responsabilidad social empresarial -RSE- se ha venido consolidando como un marco institucional que ha tenido transformaciones ligadas al desarrollo de programas que buscan que las empresas diseñen e implementen instrumentos que faciliten beneficios sociales más allá de un esquema de generación y transformación de productos. La existencia de un marco institucional para el desarrollo de 


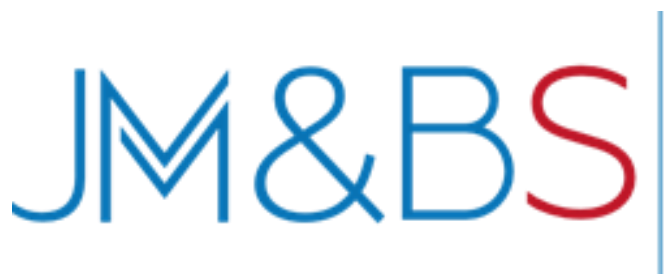

programas de RSE, no implica la obligatoriedad de las empresas de comprometerse con mejores prácticas en este campo. Según Berliner y Prakas (2014), si la RSE impone costos, entonces se puede esperar que las empresas busquen algunos beneficios al implementarla. $\mathrm{Si}$ bien algunas empresas realizan actividades de caridad, dado el contexto estructural en el que la mayoría opera esto no refleja el comportamiento de una empresa típica. Con todo, no debe confundirse las actividades caritativas con políticas de RSE.

Comúnmente, las empresas que introducen por iniciativa propia o por factores externos políticas de RSE, persiguen un posicionamiento asociado a un benchmarking que les permite reconocimiento en el mercado y beneficios estatales.

Dentro del marco institucional que regula la RSE se destaca el Pacto Global de las Naciones Unidas como un instrumento cuya finalidad es que aquellas empresas que se adhieren voluntariamente contribuyan a la construcción de un mercado global, estable, equitativo e incluyente para lograr una sociedad prospera. (Red Pacto Global Colombia, 2014).

El Pacto está integrado por componentes: derechos humanos, estándares laborales, medio ambiente y anticorrupción; que a su vez agrupan diez principios universalmente aceptados. Desde el punto de vista operativo funciona como una red integrada de trabajo donde las compañías participantes, la ONU, las ONGs y las uniones sindicales internacionales, promueven sus principios en torno a cuatro acciones: Desarrollo de iniciativas conjuntas; creación de redes de colaboración entre el mercado y la sociedad en las materias objeto del Pacto; promoción de un foro activo de educación y aprendizaje; y creación de redes y plataformas locales y nacionales (Ibarra, 2014, p. 62).

El componente de Derechos Humanos que recoge los principios del Pacto Global tiene su fundamento en la Carta Internacional de los Derechos Humanos que comprende la Declaración Universal de Derechos Humanos, el Pacto Internacional de Derechos Económicos, Sociales y Culturales, el Pacto Internacional de Derechos Civiles y 


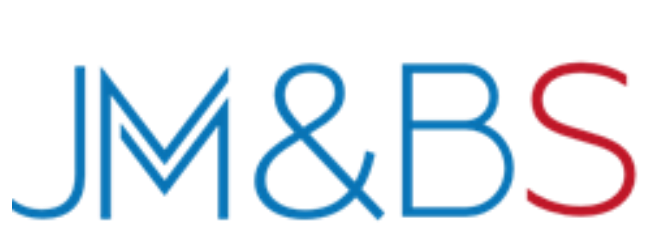

Políticos y sus dos protocolos facultativos (Red Pacto Mundial España, 2013).

Los derechos humanos en el contexto corporativo son un verdadero reto, toda vez que se trata de una obligación que en principio está orientada a la responsabilidad de los Estados. Sin embargo, se evidencia la necesidad de analizarlos desde una perspectiva empresarial (Pacto Global Colombia, 2014).

Por su parte, el componente de Derechos Laborales deriva de la Declaración de la Organización Internacional del Trabajo relativa a los principios y derechos fundamentales en el trabajo. La Declaración tiene como finalidad que los Estados miembros promuevan el respeto por los siguientes principios: la libertad de asociación, la libertad sindical y el reconocimiento efectivo del derecho de negociación colectiva; la eliminación del trabajo forzoso u obligatorio; la abolición del trabajo infantil y la eliminación de la discriminación en materia de empleo y ocupación. El Pacto Global de las Naciones Unidas los promueve como valores universales rectores de las transacciones comerciales en todo el mundo (OIT, 2014).

En relación al componente de Medio Ambiente, se exhorta a las organizaciones a cumplir con el deber de responder por los impactos ambientales que ocasionan. Para el cumplimiento de este objetivo, las organizaciones cuentan con dos enfoques: un enfoque preventivo y otro correctivo. El enfoque preventivo consiste en no ocasionar daño al medio ambiente y el enfoque correctivo pretende compensar el daño causado y los errores cometidos que lo han afectado. (Vélez, Ramírez y Rojas, 2013, p. 67).

Respecto al componente de Lucha Anticorrupción, se hace un llamado no solamente a evitar el soborno, la extorsión y otras formas de corrupción, sino también a desarrollar políticas y programas concretos que aborden el tema. Se les plantea a las empresas el reto de unirse a los gobiernos, agencias de la ONU y a la sociedad civil por una economía global más transparente (Pacto Mundial, 2013).

En el escenario comercial actual colombiano se encuentran vigentes los TLC con Estados Unidos y Canadá, por tanto el presente estudio se centra en 


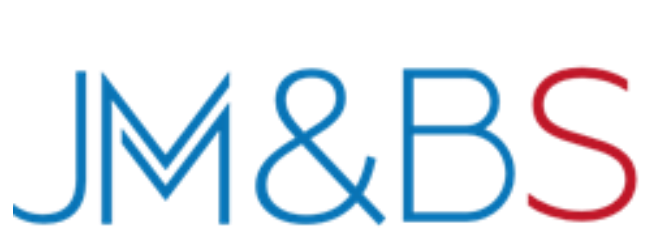

identificar el grado de compromiso de una muestra representativa de grandes empresas ubicadas en la ciudad de Barranquilla a partir de la implementación de políticas de responsabilidad social empresarial.

Específicamente, el objetivo de este trabajo es analizar las prácticas de responsabilidad social empresarial vinculadas a los diez principios del pacto global, empleadas por las empresas seleccionadas en el normal curso de sus actividades comerciales. La pertinencia de este estudio, que hace parte de una investigación mayor, gira entorno a las nuevas exigencias de la agenda comercial internacional que no solo se limita a la finalidad empresarial de consecución de utilidades privadas, sino que demanda para las empresas en trabajar en pro de los objetivos comunes de desarrollo.

El presente trabajo está organizado en tres partes: la primera presenta la metodología y el instrumento implementado para desarrollar la investigación; la segunda plantea los resultados obtenidos a raíz de la aplicación del instrumento metodológico; y la tercera expone las conclusiones por cada uno de los componentes del Pacto Global.

\section{Metodología}

La investigación parte de un enfoque mixto con predominio en lo cualitativo, que contó en este enfoque con un "proceso no matemático de interpretación, realizado con el propósito de descubrir conceptos y relaciones en los datos brutos y luego organizarlos en un esquema explicativo teórico" (Strauss y Corbin, 2002, p.12). Igualmente se presenta la construcción de teorías o categorías desde la realidad misma que se estudió (Galeano y Aristizabal, 2008, p. 23); con la medición de variables a partir del diseño de instrumentos y la interpretación de datos estadísticos, realizando generalizaciones desde los sujetos muestrales.

Luego en una segunda fase, se trató de una investigación no experimental, basada en métodos que describen las relaciones entre variables y no establecen, ni pueden probar, relaciones causales entre variables (Salkind, 1998, p. 10). Por su parte, se puede categorizar como una 


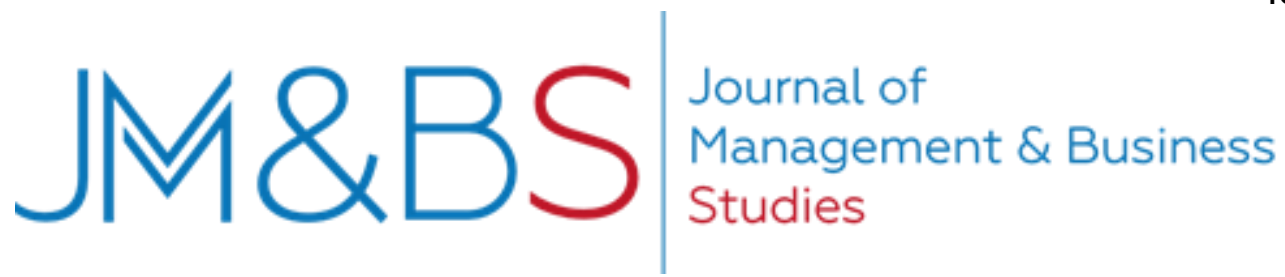

investigación de tipo exploratoriodescriptivo, ya que no solo abordó un asunto poco estudiado, teniendo en cuenta su novedad y actualidad, sino que además logró reseñar las características de la realidad sobre la responsabilidad social en Barranquilla a partir de la firma de Tratados de Libre Comercio con Estados Unidos y con Canadá.

Las técnicas e instrumentos metodológicos utilizados fueron los siguientes:

- $\quad$ En un primer lugar, para alcanzar el objetivo de establecer que tan vinculante es la normatividad sobre prácticas de responsabilidad social que surgen de los Tratados de Libre Comercio firmados entre Colombia y los Estados Unidos y Canadá, se hizo una revisión documental de la normativa y su relación que con los estándares que plantea la responsabilidad social, teniendo como punto de partida los principios del Pacto Global.

- $\quad$ En segundo lugar, se diseñó un instrumento conformado por cincuenta y seis preguntas propias de una entrevista semiestructurada, que se aplicó entre febrero y septiembre de 2015 a una muestra de las grandes empresas de la ciudad de Barranquilla pertenecientes a diferentes sectores económicos. El instrumento buscó identificar las prácticas de responsabilidad social ligadas al pacto global que surgen de los Tratados de Libre Comercio suscritos por Colombia con Estados Unidos y Canadá, a partir de la siguiente formula:

$$
\frac{n=N}{E^{2}(N-1)+1}
$$

Donde $\mathrm{N}$ equivale al universo que corresponde a las grandes empresas que exportan a Estados Unidos y Canadá; E significa el error muestral, se consideró en un $10 \%$.

Para la aplicación de la entrevista, 133 se definió la muestra a partir de un grupo de grandes empresas exportadoras a Canadá y Estados Unidos, registradas ante la Cámara de Comercio de Barranquilla. La información recopilada, fue confrontada con los informes de sostenibilidad de dichas empresas. Las empresas a las cuales se les aplicó el instrumento pertenecen a distintos sectores productivos, tales como: Comercio, farmacéutico, minero-energético e industrial.

Principalmente, el objeto de estudio de la investigación se centró en las 


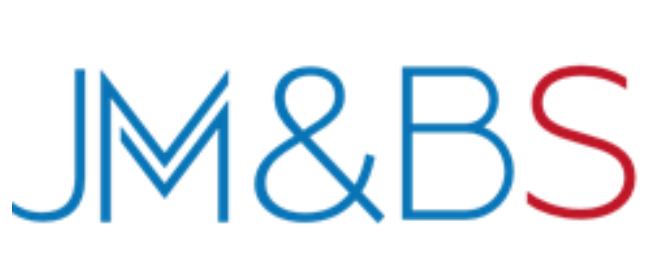

grandes empresas de la ciudad de Barranquilla. Sin embargo, se extendió la aplicación del instrumento hacia la realidad en las ciudades de Bogotá y Medellín, con la finalidad de verificar el alcance de la incidencia de los TLCs en las prácticas de Responsabilidad Social Empresarial en las grandes empresas de Barranquilla, respecto de estas otras ciudades. Finalmente, el instrumento se aplicó a dos empresas de Bogotá y dos empresas de Medellín.

- Observación no participante. Consistió en la observación de los acontecimientos y prácticas llevadas a cabo en una muestra de empresas tanto para el grupo de investigación como para el de control. Estas observaciones se realizaron teniendo como punto de partida la información obtenida dentro de las entrevistas estructuradas practicadas, a manera de confirmación o refutación de los datos obtenidos.

El procesamiento de la información, basado en el documento

6 DIAN, Dirección de impuestos y aduanas nacionales de Colombia; Superintendencia de Industria y comercio; Cámara de Comercio Colombo Canadiense; Cámara de Comercio
Spiggle (1994), se realizó partiendo de las inferencias de acuerdo con la categorización y clasificación de las respuestas, que permitieron comparar los aspectos que se abordan en el instrumento diseñado para tal fin. A los diez principios del Pacto Global, se le asignaron categorizaciones sobre los cambios que posiblemente se presentarían a raíz de la puesta en marcha delos tratados de libre comercio.

Por otro lado, también se aplicó el instrumento utilizado con las empresas, en versión sucinta, a algunas entidades ${ }^{6}$ que ejercen vigilancia y/o control de las actividades que se relacionan con la aplicación de los TLCs, y con aquellas que promueven iniciativas de responsabilidad social.

\subsection{Categorización de principios del Pacto Global}

Respecto de cada principio, se codificaron los componentes claves y se establecieron unos rangos dimensionales

Colombo Americana; Red Pacto Global Colombia; BANCOLDEX: Banco de Desarrollo empresarial; Ministerio de trabajo. 


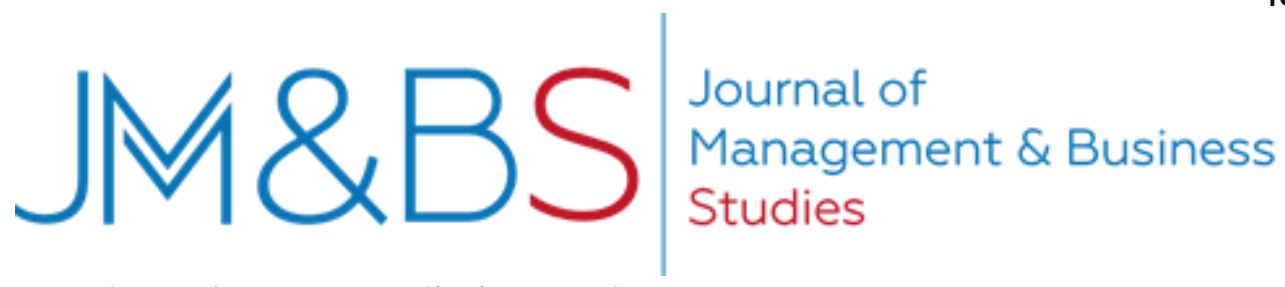

para determinar su cumplimiento, tal como

se muestra en la tabla 1:

Tabla 1. Categorización

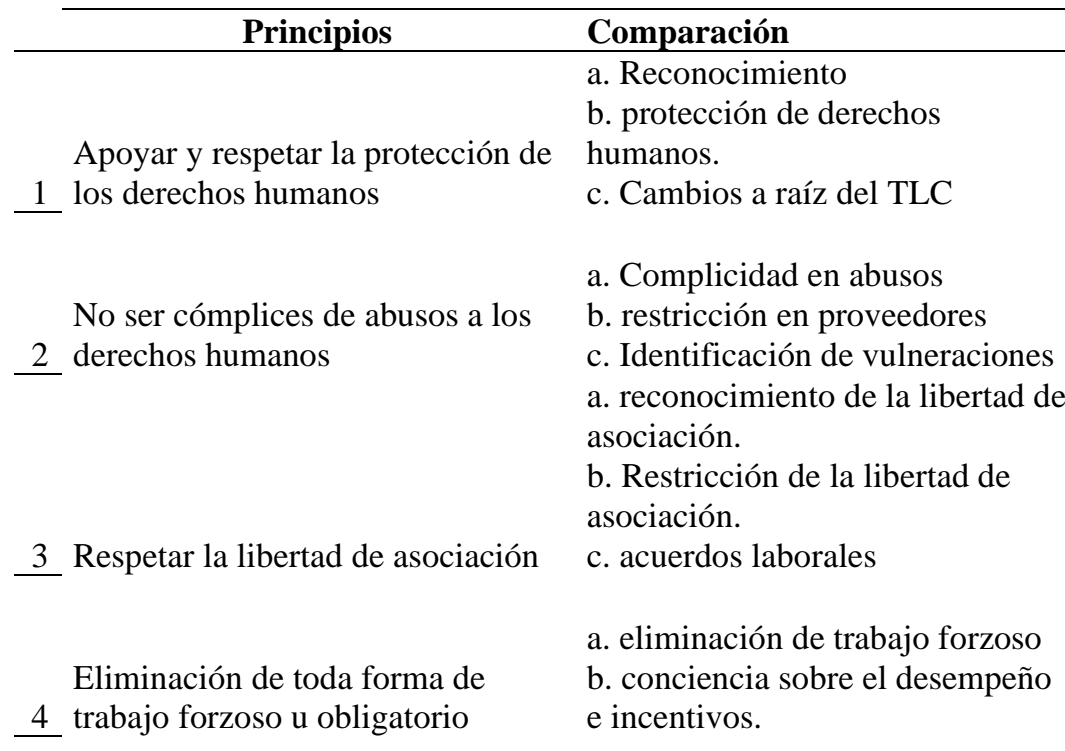

Abolición efectiva del trabajo 5 infantil

a. eliminación del trabajo infantil. Rango Dimensional

Existen:
a. $\mathrm{Si}$
b. No
c. No Informa

Existen:
a. $\mathrm{Si}$
b. No
c. No Informa

Existen:
a. $\mathrm{Si}$
b. No
c. No Informa

Existen:
a. $\mathrm{Si}$
b. No
c. No Informa

Existen:
a. $\mathrm{Si}$
b. No
c. No Informa

Existen:

a. identificación de discriminación

a. $\mathrm{Si}$

laboral.

Eliminación de la discriminación

b. selección transparente

a. identificación de riesgos ambientales.

b. No

c. No Informa

Existen:

b. cambios normativos a raíz de los TLC.

a. cumplimiento de las normas técnicas.

Promover una mayor

8 responsabilidad ambiental

b. procesos responsables con el ambiente.
a. $\mathrm{Si}$
b. No
c. No Informa

Existen:
a. $\mathrm{Si}$
b. No
c. No Informa

Existen:

a. Implementación de tecnologías
a. $\mathrm{Si}$
b. No
c. No Informa

a. identificación de riesgos sobre corrupción

Existen:
a. $\mathrm{Si}$
b. No
c. No Informa

0 corrupción procesos

Nota: Construcción propia 


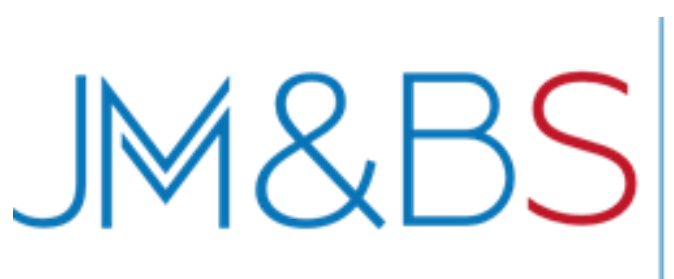

3. Resultados

Frente al componente de Derechos

Humanos el primer principio señala que las empresas deben apoyar y respetar la protección de los derechos humanos internacionalmente reconocidos. En este sentido, se indagó en los tratados de libre comercio, sobre aspectos relacionados con la protección de los derechos humanos. Del grupo de empresas a las cuales se entrevistó, el $90 \%$ coincide en que no se percibe ningún cambio a raíz de los TLCs, y que aún es muy pronto para determinar si existe o no algún tipo de influencia. Esta afirmación es compatible con la percepción de funcionarios de entidades como la Red Pacto Global Colombia, la Cámara de Comercio Colombo Americana y Colombo Canadiense.

El 40\% de las empresas afirma que se realizan actividades de promoción y reconocimiento de derechos, no solo con los trabajadores, sino con las comunidades cercanas y vulnerables.

Estas empresas manifiestan que a través de los comités de convivencia laboral promueven los derechos a nivel interno y externo para evitar vulneraciones que afectarían el funcionamiento de la empresa o a los trabajadores. Según estas, la creación de estos comités, corresponde a medidas preventivas que tienen la finalidad de promover un excelente ambiente de convivencia laboral, fomentando relaciones positivas entre los trabajadores de la empresa, así como el respaldo de su dignidad e integridad.

Según lo expresado por las empresas, en el desarrollo de sus actividades se han identificado vulneraciones de los derechos humanos a través de los mencionados comités y del análisis de riesgo realizado a los proveedores. No obstante, para los 136 investigadores fue imposible conocer casos concretos en donde se encuentren en riesgo los derechos humanos de la comunidad y de los trabajadores que podrían afectar el funcionamiento de la empresa.

El 20\% de las empresas aseguró que tiene conocimiento que en otras compañías del sector se utilizan estrategias para la identificación de riesgos en derechos humanos, el porcentaje restante manifiesta no poseer información o desconocer la implementación de 


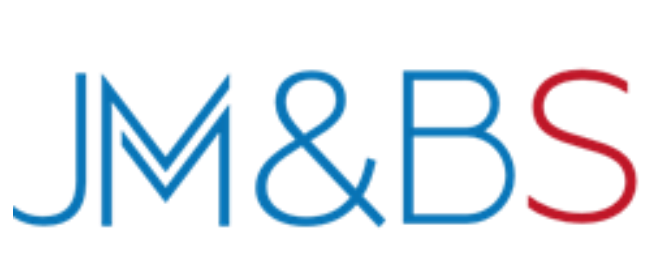

estrategias realizadas por otras empresas en ese sentido.

Respecto al segundo principio del Pacto Global que hace referencia a no ser cómplices de abusos a los derechos humanos, las empresas señalan que cuentan con un esquema de evaluación a proveedores con la finalidad de realizar seguimiento e identificación de derechos humanos en las prácticas que éstos ejecuten. Para ello cuentan con herramientas de verificación a la cadena de proveedores y socios, utilizando una matriz de riesgos donde se efectúan controles constantes que se vienen realizando, incluso con anterioridad a la entrada en vigor de los tratados de libre comercio. Algunos de los mecanismos más comunes de vigilancia utilizados, corresponden a evaluación de procesos, información sobre transparencia y trayectoria de las empresas.

Con relación al componente de derechos laborales, la muestra utilizada como base en esta investigación, señala que específicamente no se evalúa lo relacionado con los derechos humanos de los trabajadores. Sin embargo, manifiestan contar con otro tipo de herramientas como la evaluación C3 Clima y Cultura, y la evaluación de riesgo psicosocial. Ahora bien, respecto al conocimiento de prácticas implementadas por otras empresas para la identificación de garantías laborales, el $40 \%$ de la muestra afirma desconocer si éstas cuentan con estrategias en ese sentido, tal y como se refleja en la figura 1: 
ISSN 2452-5340

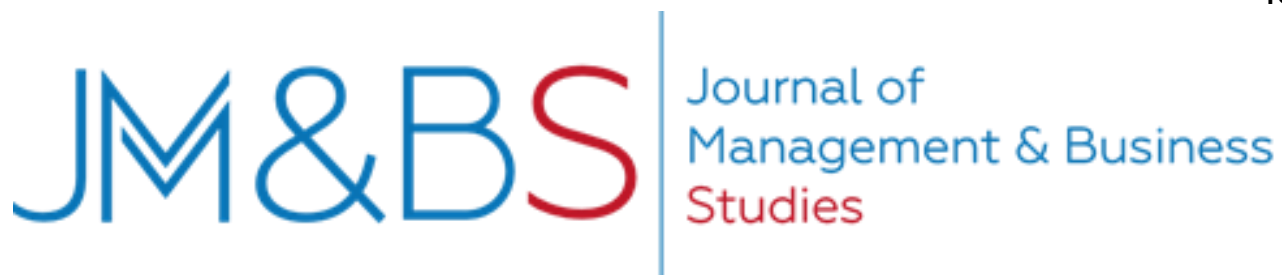

Figura 1. Respuesta de empresas entrevistadas. ¿Conoce de empresas del sector que utilicen estrategias para la identificación de derechos sociales y laborales?

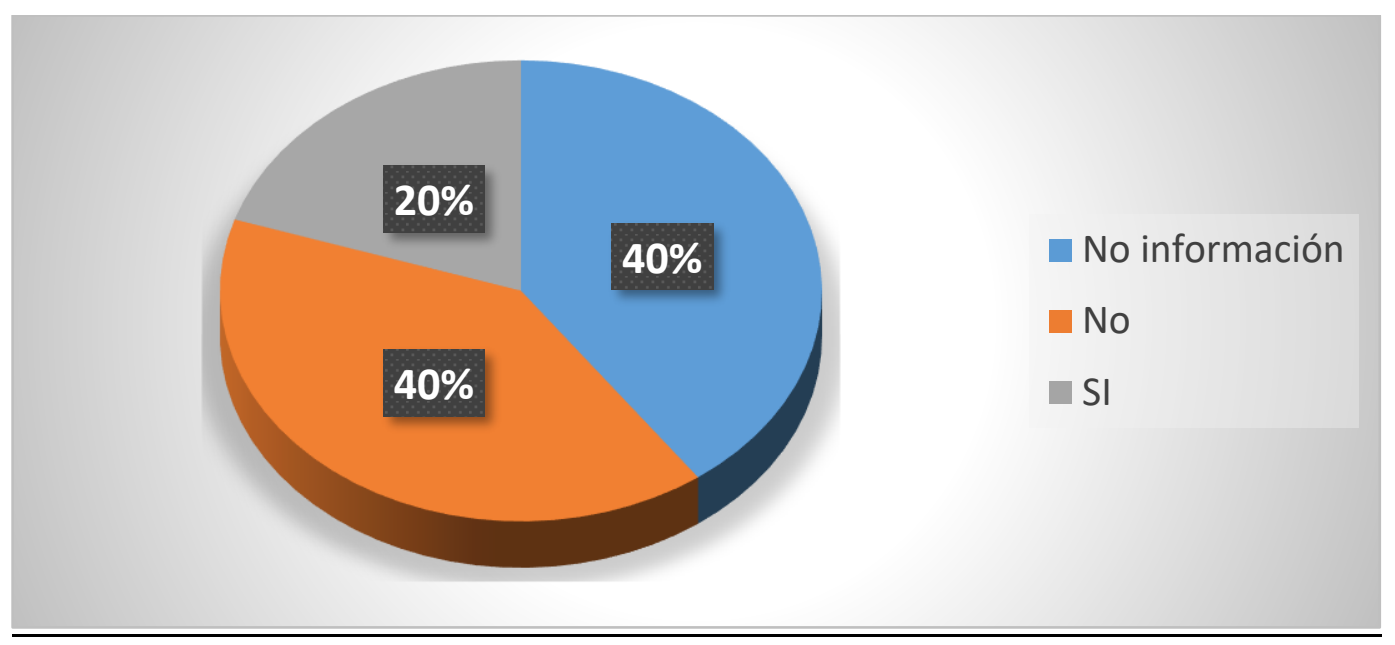

Fuente: Elaboración propia.

Frente a la promoción por parte de las empresas de la libertad de asociación sindical, el $80 \%$ de las éstas considera que las organizaciones sindicales influyen positivamente en la productividad de la empresa. Mientras que el $20 \%$ de las empresas advierte que la influencia es negativa, dado que se han presentado tensiones generadas por los trabajadores que se encuentran sindicalizados. De cualquier forma se pudo identificar no existen instrumentos o mecanismos específicos dirigidos a la garantía real y efectiva de este derecho fundamental.

Otro de los principios que enmarcan este componente invita a las empresas a apoyar la eliminación de toda forma de trabajo forzoso $\mathrm{u}$ obligatorio. Sobre el particular, el $80 \%$ de la muestra afirma expresamente que sus trabajadores conocen los términos y condiciones pactados al momento de suscribir los contratos de trabajo y cuando deciden vincularse lo hacen de forma voluntaria, siendo ésta la única práctica que las empresas asocian con este principio del pacto global.

Siguiendo con la línea de protección a las garantías laborales, el Pacto Global hace un llamado a las empresas para que apoyen la abolición efectiva del trabajo infantil. En pro de este 


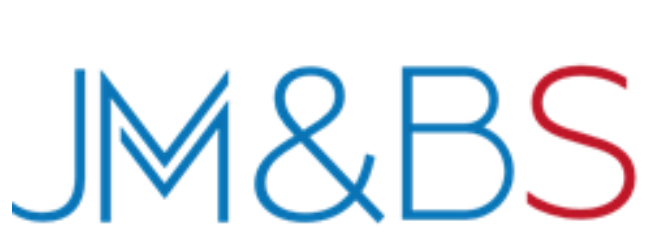

principio, el 100\% de la muestra coincidió en la importancia que representa para la sociedad el cumplimiento de este estándar internacional, y desde el punto de vista jurídico manifiestan de manera unánime conocer la prohibición establecida en la legislación colombiana al respecto. No obstante únicamente en el $20 \%$ de las empresas, se detectaron evidencias de campañas realizadas para la fomentar la abolición del trabajo y la explotación infantil.

Finalmente, el componente que venimos desarrollando incluye como principio la eliminación de la discriminación en materia de empleo y ocupación. Las empresas entrevistadas afirman que no existe discriminación en la selección de personal, puesto que vienen trabajando arduamente en la adopción de mecanismos de selección que garanticen la transparencia en los procesos de vinculación. Así las cosas, el $80 \%$ de éstas cuentan con organismos, como los comités de bienestar organizacional, que buscan minimizar el riesgo de discriminación a nivel interno.

El tercer componente abordado por el Pacto Global gira entorno a la protección del medio ambiente por parte de las empresas. Sobre el particular la totalidad de la muestra asegura que cuentan con una política empresarial que va encaminada a la protección del medio ambiente y el uso eficiente de los recursos. Para esto se efectúan estudios de impacto ambiental con el fin de realizar seguimiento y control a los posibles daños que se generarían a partir de sus actividades, en concordancia con la normativa colombiana al respecto. El $100 \%$ de las empresas han adoptado estrategias para optimizar sus recursos, disminuir contaminantes, y reutilizar 139 materiales, como se refleja en sus informes de sostenibilidad.

Las compañías encuestadas reconocieron que sus procesos industriales, tienen algunas etapas críticas que pueden causar altos impactos ambientales y para esto cuentan con una política de control y procesamiento de los residuos sólidos acorde con la ley colombiana.

Se deriva de uno de los principios que hace parte de este componente, el compromiso de las empresas de asumir iniciativas para promover una mayor 


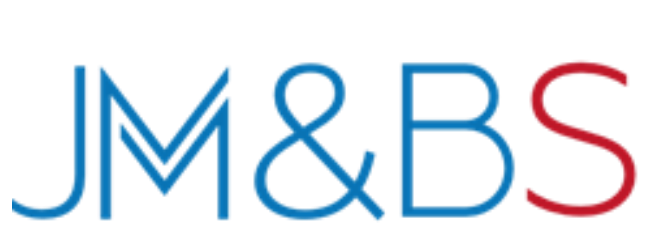

responsabilidad ambiental. En primer lugar, en términos de seguridad ambiental las empresas sostienen que el proceso y manejo de sus productos están sometidos a vigilancia constante, a través del sistema de gestión de residuos y controles en la calidad del aire. De tal suerte que el $80 \%$ de éstas califican sus productos como amigables con el ambiente, y así lo indican en sus informes a los socios comerciales y potenciales consumidores. En segundo lugar el mismo porcentaje de la muestra aseguró que realizan capacitaciones y campañas internas para promover la conciencia ambiental en sus trabajadores

Otro de los principios del Pacto Global invita a las empresas a favorecer el uso de tecnologías amigables con el medio ambiente. El 100\% de las compañías encuestadas sostienen tener un alto conocimiento de las tecnologías limpias con el ambiente que contribuyen a la eficiencia de los procesos y realizan seguimiento constante de las mejoras técnicas, así como cambios y actualizaciones en los equipos para su implementación.

El último componente que aborda el Pacto Global reivindica la implementación de políticas de anticorrupción al interior de las empresas. La totalidad de la muestra coincide en que los procesos con mayor susceptibilidad de corrupción, son en su mayoría la compra de suministros y los procesos de negociación internacional. En la misma línea el 20\% percibe que a partir de los constantes cambios en la normatividad, principalmente en el área ambiental, podrían incidir en prácticas censurables como sobornos o comisiones a funcionarios públicos

A su vez, frente a la implementación de mecanismos que 140 permitan garantizar la consecución de este principio, el $60 \%$ de las empresas cuenta con una matriz de riesgo de evaluación y descentralización de los procesos, para prevenir que sus trabajadores se involucren en actividades corruptas. Así mismo el $80 \%$ de las empresas indica que existe una política para prevenir actos de corrupción y soborno, haciendo hincapié en la existencia de comités de ética empresarial en el cumplimiento de las normas.

Las medidas señaladas por las empresas para corregir vulneraciones, son 


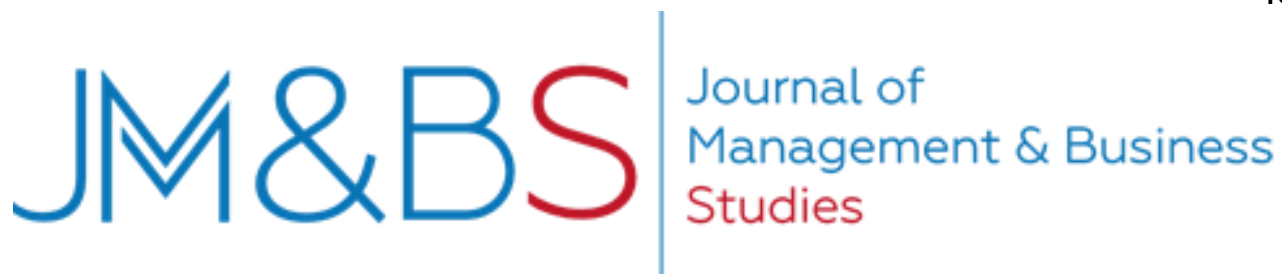

de tipo administrativo y penal

contemplados en la ley para sancionar dependiendo del grado de vulneración. El $40 \%$ de éstas manifiesta que recientemente se han visto expuestas a presiones e incluso propuestas poco éticas o de dudosa legalidad por parte de proveedores $\mathrm{y}$ socios comerciales. Frente a este hecho, las empresas han optado por recurrir a los mecanismos legalmente establecidos dichas conductas.

Tal y como se enuncia en el acápite de metodología, a continuación, en la tabla 2 se presentan los resultados del análisis derivado de la codificación realizada a cada uno de los componentes del Pacto Global de conformidad con sus principios y porcentaje de cumplimiento:

Tabla 2. Consolidado de puntaje promedio de cumplimiento de los principios.

\begin{tabular}{|c|c|c|}
\hline Componentes & Principios & Porcentaje \\
\hline \multirow[t]{2}{*}{$\begin{array}{l}\text { Derechos } \\
\text { Humanos }\end{array}$} & $\begin{array}{l}\text { Principio No. 1: Las empresas deben apoyar y respetar la } \\
\text { protección de los derechos humanos reconocidos a nivel } \\
\text { internacional. }\end{array}$ & $70 \%$ \\
\hline & $\begin{array}{l}\text { Principio No. 2: Las empresas deben asegurarse de no ser } \\
\text { cómplices de abusos a los derechos humanos }\end{array}$ & $67 \%$ \\
\hline \multirow[t]{4}{*}{$\begin{array}{l}\text { Derechos } \\
\text { Laborales }\end{array}$} & $\begin{array}{l}\text { Principio No. 3: Las empresas deben respetar la libertad de } \\
\text { asociación sindical y el reconocimiento efectivo del derecho a } \\
\text { la negociación colectiva }\end{array}$ & $60 \%$ \\
\hline & $\begin{array}{l}\text { Principio No. 4: Las empresas deben apoyar la eliminación } \\
\text { de toda forma de trabajo forzoso u obligatorio }\end{array}$ & $95 \%$ \\
\hline & $\begin{array}{l}\text { Principio No. 5: Las empresas deben apoyar la abolición } \\
\text { efectiva del trabajo infantil }\end{array}$ & $100 \%$ \\
\hline & $\begin{array}{l}\text { Principio 6: Promover la eliminación de la discriminación en } \\
\text { materia de empleo y ocupación }\end{array}$ & $96 \%$ \\
\hline \multirow[t]{3}{*}{$\begin{array}{c}\text { Medio } \\
\text { Ambiente }\end{array}$} & $\begin{array}{l}\text { Principio 7: Apoyar la aplicación de un criterio de } \\
\text { precaución respecto de los problemas ambientales }\end{array}$ & $95 \%$ \\
\hline & $\begin{array}{l}\text { Principio 8: Adoptar iniciativas para promover una mayor } \\
\text { responsabilidad ambiental }\end{array}$ & $94 \%$ \\
\hline & $\begin{array}{l}\text { Principio 9: Alentar el desarrollo y la difusión de tecnologías } \\
\text { inocuas para el medio ambiente }\end{array}$ & $94 \%$ \\
\hline Anticorrupción & $\begin{array}{l}\text { Principio 10: Las empresas deben actuar contra todas las } \\
\text { formas de corrupción, incluyendo la extorsión y el soborno. }\end{array}$ & $90 \%$ \\
\hline
\end{tabular}

Fuente: Elaboración propia.

Promedio General | $\mathbf{8 6 \%}$ 
ISSN 2452-5340

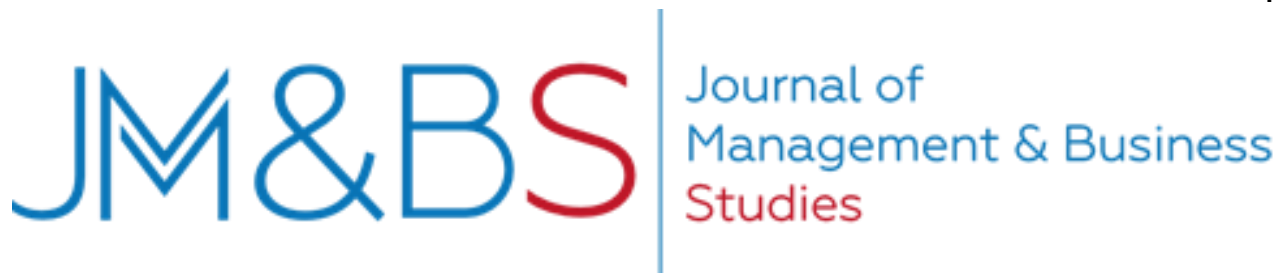

Del análisis de los datos que se reflejan en respecto de los componentes que la tabla No. 2 se evidencia el porcentaje de contempla el Pacto Global, tal y como se desempeño que las empresas han tenido observa en la figura. 2.

Figura 2. Desempeño de las empresas frente al Pacto Global.
D.H
$68 \%$
D.L
$88 \%$
$94 \%$
$90 \%$
M.A
A.C

Fuente: Elaboración propia.

Así las cosas, la implementación de políticas de anticorrupción y de medio ambiente se destacan con un $90 \%$ y $94 \%$ respectivamente. Respecto de los derechos laborales se observa un desempeño del $88 \%$, mientras que el porcentaje más bajo hace relación al componente de derechos humanos con un $68 \%$.

\section{Conclusiones}

Luego de aplicada la entrevista y analizados los resultados, frente a la creación de prácticas en materia de Responsabilidad social relacionadas con el componente de Derechos Humanos del Pacto Global, el 100\% de la muestra manifestó no contar con estructuras internas, llámense departamentos o divisiones, que tengan como finalidad exigir el respeto de garantías y libertades fundamentales. Esto no genera violaciones directas a los derechos humanos, pero si podría propiciar su irrespeto, puesto que al no contar con este tipo de áreas al interior de la organización, quienes viesen afectados sus derechos por el actuar empresarial, no tendrían a quien recurrir en primera medida.

Sin duda constituye un gran avance en términos jurídicos, que las empresas privadas se encuentren cobijadas por las disposiciones de la Declaración Universal 


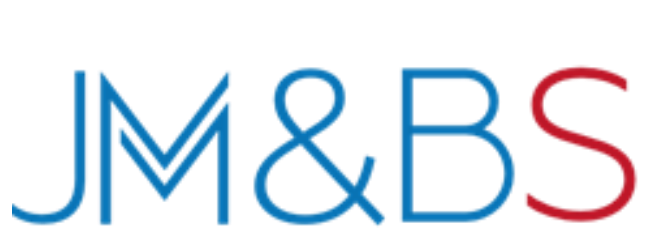

de los Derechos Humanos, puesto que se pretende cambiar de paradigma. Dicho cambio consiste en hacer el tránsito de una postura clásica o realista, en la que el único sujeto que puede violar derechos humanos es el Estado, hacia una postura moderna o liberalista, en la que se contempla, además del Estado, a las empresas como actores que podrían vulnerar estos derechos.

Se resalta que los medios más utilizados en las empresas para verificar si se está vulnerando algún tipo de derecho, a nivel interno o externo ${ }^{7}$, que afecten el funcionamiento de la empresa o a los trabajadores, corresponden a los comités de convivencia laboral ${ }^{8}$. Sin embargo, la competencia de dichos comités se encuentra doblemente limitada: en primer lugar, se limita a los derechos laborales frente al resto de derechos humanos; en segundo lugar, se limita a la convivencia laboral frente al universo de libertades jurídicas del mundo del trabajo. Es decir,

\footnotetext{
${ }^{7}$ Cuando se habla de vulneraciones a nivel interno o externo, nos referimos básicamente al ámbito de acción de la empresa. En este sentido, las violaciones internas a los derechos humanos se refieren al área de control de las empresas, esto es, a sus trabajadores, contratistas y similares. A su vez, las violaciones externas tienen que ver con el área de influencia, esto es, todas las vulneraciones cometidas por la empresa que no tengan relación
}

no se identifica claramente una estructura al interior de las empresas que promueva y promocione el respeto y la protección de los derechos Humanos. Sumado a esto, si bien el $40 \%$ de la muestra ha logrado identificar vulneraciones de los derechos a través de los comités, no fue posible determinar vulneraciones a los derechos a nivel interno o externo que afecten el funcionamiento de la empresa o a los trabajadores. Lo anterior demuestra que además de que no existen estructuras claras de defensa y protección de los derechos Humanos al interior de las empresas, los esfuerzos representados en los comités de convivencia laboral son insuficientes.

En este mismo sentido, al indagar el instrumento por mecanismos de respeto a los Derechos Humanos, las empresas señalaban herramientas tales como: reglamentos internos de trabajo, códigos de ética, evaluaciones de riesgo

con el área de control, por ejemplo: violaciones a los derechos de la comunidad y al medio ambiente. ${ }^{8}$ Los Comités de Convivencia Laboral “(...) son una medida preventiva que tienen la finalidad de promover un excelente ambiente de convivencia laboral, fomentar relaciones positivas entre los trabajadores de la empresa, y respaldar la dignidad e integridad de las personas en el trabajo" (Ministerio del Trabajo, Resolución 1356, 2012) 


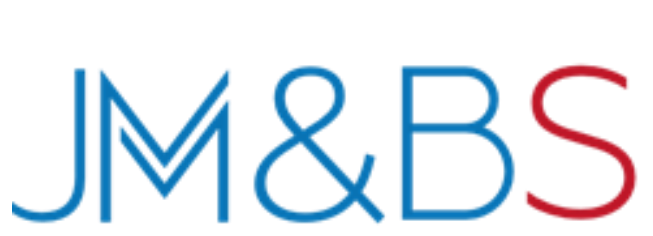

psicosocial, evaluaciones sobre clima y cultura. Ahora bien, si no se conocen cuáles son las herramientas y mecanismos idóneos para implementar estructuras internas que busquen el respeto de los Derechos Humanos, estos se ponen en riesgo de forma automática puesto que se crea una barrera, basada en el desconocimiento, para el reconocimiento y la promoción de los mismos.

En este estado surge una inconsistencia al comparar los resultados encontrados por el instrumento de investigación con los Principios Rectores sobre las empresas y los derechos humanos: puesta en práctica del marco de las Naciones Unidas para "proteger, respetar y remediar". Al respecto el Principio 15 señala que "para cumplir con su responsabilidad de respetar los derechos humanos, las empresas deben contar con políticas y procedimientos apropiados en función de su tamaño y circunstancias (...)". Los comités de convivencia laboral, códigos de ética, evaluaciones de riesgo psicosocial y demás mecanismos aplicados por las empresas de la muestra no cumplen con las características establecidas en el mencionado principio, esto es:

$$
\text { “(...) a) Un compromiso }
$$

político de asumir su responsabilidad de respetar los Derechos Humanos; b) Un proceso de diligencia debida en materia de derechos humanos para identificar, prevenir, mitigar y rendir cuentas de cómo abordan su impacto sobre los derechos humanos; c) Unos procesos que permitan reparar todas las consecuencias negativas sobre los derechos humanos que hayan provocado o contribuido a provocar" (Consejo de Derechos Humanos-Naciones Unidas, 2011) El respeto a los Derechos Humanos por parte de las empresas debe partir de un compromiso político y procedimientos adecuados que desarrollen la debida diligencia, prevengan las vulneraciones y reparen las consecuencias negativas del actuar de la empresa. Los mecanismos que manifiestan aplicar las empresas en el instrumento aplicado, resultan insuficientes frente al marco jurídico que establece el Derecho Internacional de los Derechos Humanos. El hecho que no se 


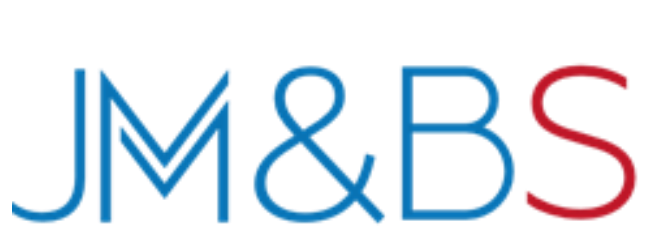

cuente con estructuras empresariales que promuevan las libertades fundamentales es un reflejo de la ausencia del Estado como principal actor en su protección de los Derechos Humanos.

El Principio 3 sobre las Empresas y

los Derechos Humanos, señala:

"En cumplimiento de su obligación de protección, los Estados deben: a) Hacer cumplir las leyes que tengan por objeto o por efecto hacer respetar los derechos humanos a las empresas, evaluar periódicamente si tales leyes resultan adecuadas $\mathrm{y}$ remediar eventuales carencias; b) Asegurar que otras leyes y normas que rigen la creación y las actividades de las empresas, como el derecho mercantil, no restrinjan sino que propicien el respeto de los derechos humanos por las empresas; c) Asesorar de manera eficaz a las empresas sobre cómo respetar los derechos humanos en sus actividades; d) Alentar y si es preciso exigir a las empresas que

${ }^{9}$ Leyes entendidas en un sentido amplio, como toda norma, instrumento o elemento que conforma el expliquen cómo tienen en cuenta el impacto de sus actividades sobre los derechos humanos." Consejo de Derechos Humanos-Naciones Unidas, 2011)

Esto es, el deber de respetar los derechos humanos, que en virtud de los Principios Ruggie recae en cabeza de las empresas, se encuentra en relación directa con la obligación de los Estados de protegerlos. Es decir, en el territorio nacional el Estado es el responsable de proteger y promocionar los derechos de todas las personas. En ejecución de dicha obligación, los estados han de “(...) hacer cumplir las leyes que tengan por objeto o por efecto hacer respetar los derechos humanos a las empresas (...)”. En relación con las empresas, las principales leyes ${ }^{9}$ que buscan el respeto de los derechos son: la Carta Internacional de los Derechos Humanos, el Pacto Global y los Principios Ruggie. De esta manera, si las empresas no cumplen, por ejemplo, con su responsabilidad de respetar los derechos humanos a través de la implementación de políticas y procedimientos, se estaría

sistema jurídico tanto a nivel nacional como internacional. 


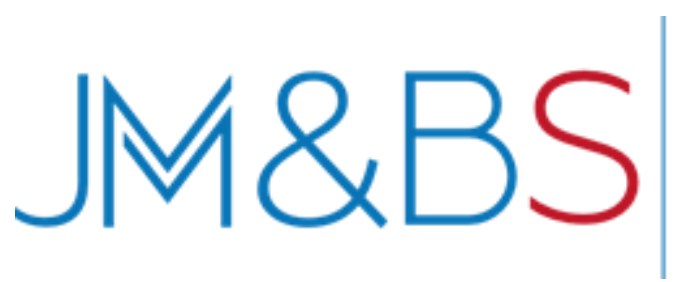

configurando una omisión del Estado frente a su obligación de protección puesto que no hizo cumplir esa Ley en especial.

Del análisis del instrumento aplicado, se destaca que las empresas tienden a confundir caridad con RSE y consideran que de esa manera están cumpliendo con sus obligaciones en materia de derechos humanos.

Así, el 20\% de las empresas señaló como parte de sus actividades en relación con la comunidad la realización de jornadas de salud, entrega de kits de sus productos, realización de jornadas de limpieza, adecuación de colegios y aulas de clases. Sin embargo, por más caritativas y filantrópicas que sean estas acciones, no constituyen un concepto de gestión de la actividad empresarial en relación con su área de influencia (comunidad) y no satisface las obligaciones de la empresa frente a los derechos humanos.

Por otra parte, con relación al componente de Derechos Laborales se pudo constatar que los acuerdos comerciales suscritos por Colombia con Canadá y Estados Unidos respectivamente mantienen el compromiso de continuar respetando las obligaciones asumidas en calidad de países miembro de la Organización Internacional del Trabajo.

En este orden de ideas, se considera que la intención de continuar reivindicando y garantizando los derechos laborales de los trabajadores en armonía con los términos de los acuerdos comerciales suscritos, es un intento por impulsar el proceso de integración de la normatividad internacional hacia los derechos laborales reconocidos por cada Estado contratante, y en particular para el desarrollo de los principios de libertad de asociación, eliminación de toda forma de trabajo forzoso u obligatorio, abolición efectiva del trabajo infantil y eliminación de la discriminación en materia de empleo y ocupación.

Así las cosas, la principal conclusión respecto del componente mencionado, es que no se observa incidencia en las políticas laborales al interior de la muestra analizada como consecuencia de la firma de los tratados de libre comercio firmado con Estados Unidos y Canadá. Incluso los representantes de la Red del Pacto Global para Colombia, y la Cámara de Comercio Colombo-Canadiense, afirmaron que hasta 


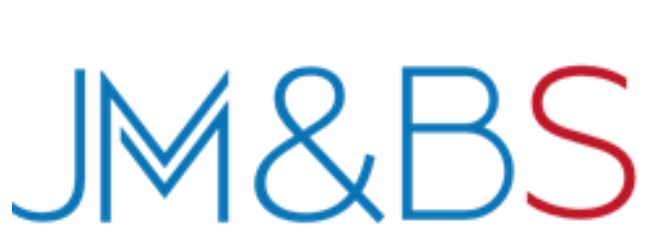

este momento no hay influencia directa de los tratados en las prácticas de las grandes empresas de Barranquilla.

En particular sobre el principio de libertad de asociación sindical, la investigación develó la resistencia por parte de los empresarios, hacia la promoción de este derecho, a pesar de que el Estado colombiano a partir de la firma de los tratados comerciales se comprometió a vigilar su cumplimiento. Incluso las asociaciones sindicales presentes, fueron creadas antes de la entrada en vigor de los acuerdos comerciales entre Colombia con Estados Unidos y con Canadá. Adicionalmente no existe influencia directa por parte de los socios comerciales para realizar cambios en sus políticas laborales a nivel interno, de tal forma que se promueva la libertad de asociación sindical.

En relación al compromiso de eliminar toda forma de trabajo forzoso y obligatorio, asumida por el Estado colombiano al suscribir los tratados de libre comercio con Estados Unidos y Canadá, de los resultados arrojados por el instrumento se evidencia un claro rechazo de la totalidad de las empresas a ejercer este tipo de prácticas. Ahora bien, el hecho de que en las empresas no se presenten situaciones relacionadas con la exclusión del trabajo forzoso $\mathrm{y}$ obligatorio no deviene de la firma de los tratados de libre comercio, sino del cumplimiento de las políticas internacionales promovidas por la OIT, ratificados por el país, y ampliamente difundidas entre los actores de la relación laboral.

Respecto del compromiso adquirido por el Estado colombiano, para eliminar el trabajo infantil, todas las empresas entrevistadas reconocen la prohibición de contratar niños o niñas en su actividad. No obstante, el hecho de que solo el $20 \%$ de éstas realicen campañas para la abolición del trabajo y la explotación infantil, lo justifican por el costo que les representa dicha inversión escapando en su criterio la órbita de los privados, por lo que únicamente se limitan a cumplir con la normativa laboral que establece edades mínimas para la contratación del talento humano.

Por su parte, frente a la eliminación de discriminación en materia de empleo y ocupación, las empresas confían en el comité de bienestar como mecanismo 


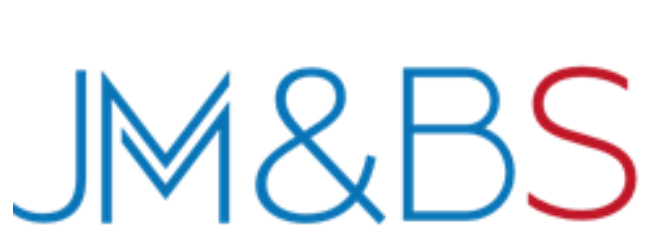

idóneo para evitar la discriminación.

Sostienen que en sus procesos de selección

y contratación del personal se abstienen de valorar criterios como la religión, identificación racial, orientación sexual, opinión política, o nacionalidad.

En definitiva, como se ha podido observar, en materia laboral no existe influencia directa ni del Pacto Global, ni de los tratados de libre comercio de Canadá y Estados Unidos con el desempeño y las dinámicas laborales que se observan al interior de las empresas grandes empresas de la muestra en Barranquilla.

Los principios de la categoría de Medio ambiente del Pacto Global, así como los tratados de libre comercio y los demás instrumentos jurídicos internacionales que contemplan disposiciones dirigidas a la protección del ambiente carecen de mecanismos que los hagan eficaces, por lo que su cumplimiento termina dependiendo de la buena voluntad de quienes en ellos se comprometen. Bien es sabido que son los Estados quienes contraen obligaciones en calidad de partes en los tratados internacionales y no las empresas a quienes se dirige el Pacto Global como principal iniciativa de RSE. De ahí que en el instrumento aplicado no se evidencie incidencia de los tratados de libre comercio en el cumplimiento de los principios del Pacto Global, en donde si bien dichos principios aparecen, no hay una mención expresa al Pacto ni como relativos a la responsabilidad social empresarial.

El carácter voluntario de la inclusión que hacen las empresas al Pacto Global y en general de adoptar comportamientos coherentes con la responsabilidad social empresarial en materia ambiental, hace que cuando el Estado, en calidad de parte, como en el caso de los TLC's aquí estudiados, sea quien los promueva, se dificulte su entendimiento y puesta en marcha por parte de los privados que realizan actividades productivas en el marco de los mismos. Máxime si en Colombia no se conocen premios ni reconocimientos estatales a las empresas por la implementación de prácticas de responsabilidad social empresarial, como si se tratara de imperativos, y que para el caso del TLC entre Colombia y Canadá, a 


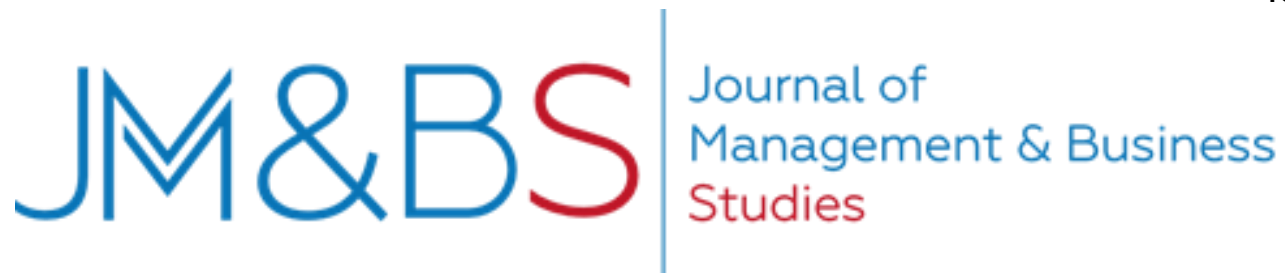

pesar de haberse suscrito un acuerdo para la conservación y protección ambiental, adicional al tratado, no se incluyeron medidas que estimulen la responsabilidad social empresarial, ni se promueva la efectiva participación ciudadana, señalándola tan sólo en términos de información y denuncia.

Lo anterior, así como el instrumento aplicado, denotan una desconexión entre el Estado y las empresas, que señalan conocer y cumplir sus obligaciones en materia ambiental derivadas de las normas nacionales, afirmando al mismo tiempo que son confusas y menos estrictas que las internacionales, siendo el cumplimiento de estas últimas, según las propias empresas, más sencillo en razón a su estabilidad y claridad.

Así como existe una desarticulación entre el Estado y la comunidad a efecto de la protección ambiental, pues en la mayoría de los casos las decisiones que lo pueden afectar se toman sin garantizar la participación efectiva de la ciudadanía, también existe desconexión entre las empresas y las poblaciones aledañas a sus centros de operación, lo que conlleva el desconocimiento por parte de las empresas de las afectaciones que pueden estar sufriendo estas comunidades y su exclusión en las políticas internas que señalan como respuesta a su preocupación por la protección del ambiente en el marco de los intereses empresariales, sociales y económicos.

A pesar de que el $80 \%$ de las empresas coincide en la necesidad de mantener un enfoque preventivo que favorezca el ambiente y el desarrollo de tecnologías más respetuosas con el mismo, en ninguna de ellas aparece el fomento a iniciativas que promuevan una mayor responsabilidad ambiental, en términos del deber de reparar los daños ambientales que puedan generar, ni una clara conciencia de la relevancia del principio del contaminador-pagado que se encuentra en la Declaración de Río sobre el medio ambiente y el desarrollo. Dentro de su política de calidad se incluye de manera separada la responsabilidad social y lo ambiental, lo que evidencia un desconocimiento o por lo menos falta de claridad de lo que este concepto integra, y cuando incluyen dentro de sus políticas el 


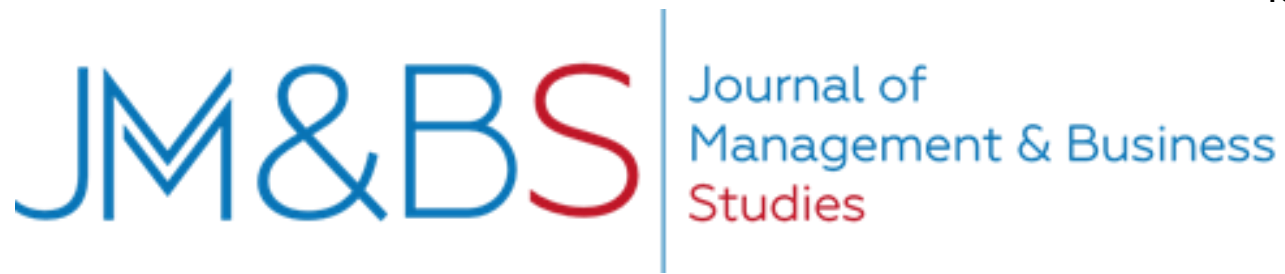

principio del desarrollo sostenible, entendido como el equilibrio entre el desarrollo de la empresa, el bienestar social y el impacto ambiental, no contemplan acciones específicas que vinculen a las comunidades aledañas a sus centros de operaciones, de tal manera que se puedan promover también con estas las prácticas de responsabilidad social empresarial.

Se destaca que en un porcentaje muy mínimo -solo el 20\%-de las empresas consultadas se pudo evidenciar una política de sostenibilidad como parte de su eje estratégico, que incluye la dimensión ambiental junto con la económica y la social, haciendo referencia expresa al Pacto Global, que se implementa a través de talleres de sensibilización y publicación de información en medios internos de comunicación, incluyendo a la comunidad tangencialmente, refiriéndose a la buena voluntad y a una estructura interna denominada como gobierno de la sostenibilidad como responsables de impulsar y ejecutar dichos propósitos.

En definitiva se observó que las grandes empresas objeto de esta investigación, más que ser socialmente responsables lo que hacen es implementar algunas prácticas que las vuelven más atractivas frente a los consumidores, resultando así en una estrategia de negocio más que en un verdadero compromiso frente al clamor social por la crisis ambiental ampliamente documentada en la que se encuentra el planeta.

En cuanto a la lucha contra la corrupción las empresas investigadas tienen compromiso y claridad frente a los riesgos que ese tipo de prácticas generan, $\mathrm{y}$ a las acciones que se deben llevar a cabo para combatirlas. De acuerdo con los resultados de esta investigación las 150 empresas conocen los espacios que son más propensos dentro de las actividades corporativas a actos de corrupción.

Frente a la percepción de prácticas corruptas en el sector privado, la tercera encuesta nacional anticorrupción (Yepes, Restrepo y Rodríguez, 2012) muestra que el $94 \%$ de los empresarios considera que en el giro normal de sus negocios las empresas acuden a sobornos. Este dato resulta paradójico comparado con los resultados de la investigación realizada, puesto que solo el $40 \%$ de las empresas de la muestra analizada afirman haberse visto 


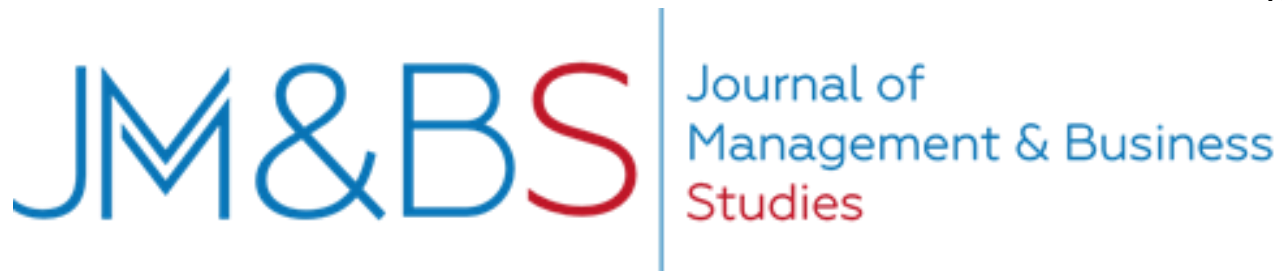

presionadas por prácticas corruptas y el 60 $\%$ sostiene que cuentan con un mapa de riesgo de corrupción identificado y mecanismos de mitigación y minimización de las áreas funcionales más susceptibles de actos de corrupción.

La mayoría de las empresas que participaron en esta investigación afirman que cuentan con herramientas para disminuir las probabilidades de corrupción, específicamente hablan del comité de ética, pero dicho instrumento es solo una pieza dentro de un sistema de gestión ética que no permite ejercer un control suficiente sobre las operaciones empresariales, por ello aun la percepción de la necesidad de sobornos y prácticas corruptas para poder seguir en los negocios es extremadamente alta. En otros casos se habla del código ética pero es claro que en muchas empresas esta herramienta solo se trata de un documento redactado que tiene poca o nula aplicación y muchas veces ni siquiera se trata de un instrumento de auditoría, sino más bien en una parte más del discurso empresarial.

La corrupción es un tema tabú, por un lado, desde los ejecutivos existe una percepción de la necesidad de pagar sobornos para poder operar, por otro lado, parece existir un consenso general en que es una práctica ilegal que hay que minimizar no solo en el contexto nacional sino también en el internacional. De hecho, desde los mecanismos multilaterales hay una necesidad de mejorar los esquemas de lucha contra la corrupción, por ello la incidencia de los TLC en la lucha contra la corrupción sí parece ser explícita en las prácticas de responsabilidad social en las grandes empresas que exportan a EE.UU y Canadá, al menos desde el discurso y las relaciones públicas empresariales.

En este punto se reitera lo ya mencionado, esto es, las empresas coinciden en su compromiso con las prácticas de Responsabilidad Social Empresarial, desde antes de la entrada en vigor de los TLCs suscritos entre Colombia con Estados Unidos y con Canadá e independientemente de su adhesión al Pacto Global.

En relación con las empresas que hacen parte del Pacto Global, se pudo establecer que tanto en sus canales de información como en sus informes de sostenibilidad aparecen claramente 
ISSN 2452-5340

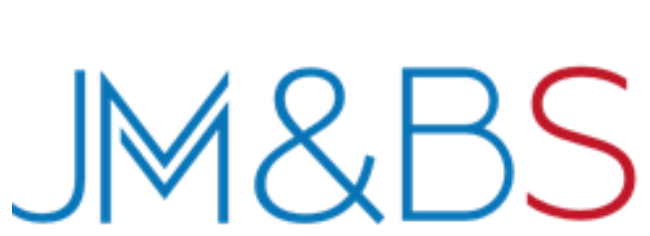

definidas las categorías de derechos humanos, derechos laborales, medio ambiente y lucha anticorrupción, así como los mecanismos para ponerlas en marcha.

El discurso de las empresas no coincide con las evidencia obtenidas en la investigación, en ninguna de las categorías de la Responsabilidad Social Empresarial a las que hace referencia el Pacto Global, en el sentido que las herramientas que utilizan las empresas son insuficientes o meramente formales, de tal manera que no producen el impacto esperado tanto al interior de las empresas como en su área de influencia.
Las empresas no conocen el alcance de los principios del Pacto Global, ni existe una sincronía entre estos y lo que conciben como prácticas de responsabilidad social empresarial.

Ha quedo establecido el discreto papel del Estado colombiano en la promoción de prácticas de Responsabilidad social empresarial y en la aplicación de los distintos instrumentos de que dispone para el cumplimiento de las obligaciones derivadas de los TLC's suscritos con Estados Unidos y con Canadá.

\section{Referencias:}

Ancos, H. y Vicente, D. (2009). La promoción de la responsabilidad social en los tratados de inversión. Los casos de Bolivia y Venezuela. España, Madrid: Fundación Carolina.

Berliner, D. \& Prakash. A. (2014).The United Nations Global Compact: An Institutionalist Perspective. Journal of Business Ethics, 122,217-223. DOI: 10.1007/s10551-014-2217-5

Galeano, M. y Aristizábal, M. (2008). Cómo se construye un sistema categorial. Revista Estudios de Derecho, Vol. 65, No. 125, 164-187.

Ibarra, A. (2011). Principios de la responsabilidad social empresarial en el ordenamiento jurídico colombiano. Tesina de maestría, Universidad del Norte

Ibarra, A. (2014). Principios de la responsabilidad social empresarial en el ordenamiento jurídico colombiano. Revista de Derecho, 41, pp. 51-82. DOI: http://dx.doi.org/10.14482/dere.41.5443.

Naciones Unidas, Consejo de Derechos Humanos (2015). Informe del Alto Comisionado de las Naciones Unidas para los Derechos Humanos sobre la situación de los derechos humanos en Colombia (A/HRC/28/3/Add.3). Recuperado de http://www.hchr.org.co/documentoseinformes/informes/altocomisionado/A_HRC_28_3 Add_3_SPA.pdf 
ISSN 2452-5340

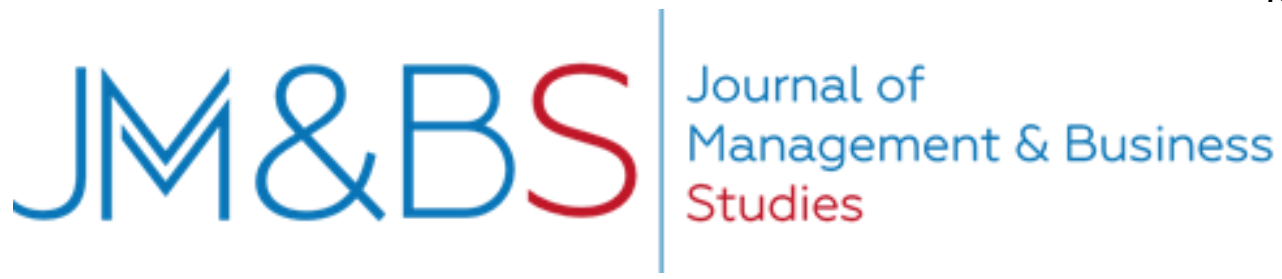

Organización Internacional del Trabajo (2014). Recuperado el 09 de febrero de 2015, de http://www.ilo.org/global/lang--es/index.htm

Red Pacto Mundial España, (2013). Recuperado el 09 de febrero de 2015, dewww.pactomundial.org

Red Argentina del Pacto Global (2004). Recuperado el 14 de mayo de 2015, de http://www.pactoglobal.org.ar/

Salkind, N. (1999). El papel y la importancia de la investigación. En Neil J Salkind (Ed.), Métodos de investigación. México: Prentice Hall

Spiggle, S. (1994). Analysis and Interpretation of Qualitative Data in Consumer Research. Journal of Consumer Research, Vol. 21, No 3, pp. 491-503

Strauss, A. y Corbin, J. (2002). Bases de la investigación cualitativa. Técnicas y procedimientos para desarrollar la teoría fundamentada. Colombia, Bogotá: CONTUSEditorial, Universidad de Antioquia.

Transparency international (2009). Informe Global de la Corrupción: 2009 Corrupción y sector privado. Recuperado de http://www.bib.ufro.cl/portalv3/files/informe-global-de-lacorrupcion.pdf

Vélez, D., Ramírez, C. y Rojas, G. (2013). Gerencia de la Responsabilidad Social en las organizaciones de hoy. Ibagué: Universidad de Ibagué.

Yepes, G., Restrepo, C. y Rodríguez, M. (2012). Colección cuadernos de transparencia. Colombia, Bogotá: Corporación Transparencia por Colombia y Universidad Externado de Colombia. DOI: 10.1086/209413 